\title{
ASYMPTOTIC DISTRIBUTION OF THE DISCOUNTED PROPER DEFICIT IN THE DISCRETE TIME DELAYED RENEWAL MODEL
}

\author{
Zhen-HUA BAO AND JING WANG
}

\begin{abstract}
In this paper we consider the discrete time delayed renewal risk model. We investigate what will happen when the distribution function of the discounted proper deficit is asymptotic in the initial surplus. In doing this we establish several lemmas regarding some related ruin quantities in the discrete time delayed renewal risk model, which are of significance on their own right.
\end{abstract}

\section{Introduction}

We begin with the ordinary discrete time renewal risk model. The individual claim amounts $\left\{X_{i}: i \in \mathbf{N}^{+}\right\}$are independent and identically distributed (i.i.d.) positive random variables with common probability function (p.f.) $f(x)$, $x \in \mathbf{N}^{+}$and distribution function (d.f.) $F(x)=1-\bar{F}(x)$. The counting process $\{N(n), n \in \mathbf{N}\}$ denotes the number of claims up to time $n$ and is defined as $N(n)=\max \left\{k: W_{1}+W_{2}+\cdots+W_{k} \leq n\right\}$, where the interclaim times $\left\{W_{i}: i \in \mathbf{N}^{+}\right\}$are i.i.d. positive random variables with common p.f. $k(t)$, $t \in \mathbf{N}^{+}$, the d.f. $K(t)=1-\bar{K}(t)$, and the mean $E\left(W_{2}\right)<\infty$. We assume that $\left\{X_{i}: i \in \mathbf{N}^{+}\right\}$and $\left\{W_{i}: i \in \mathbf{N}^{+}\right\}$are mutually independent. The surplus of the insurer at time $n$ is then defined as

$$
U(n)=u+n-\sum_{i=1}^{N(n)} X_{i}, n=1,2, \ldots,
$$

where $u \in \mathbf{N}$ is the initial surplus.

In the delayed renewal risk model, the number of claims process $\{N(n), n \in$ $\mathbf{N}\}$ is assumed to be delayed renewal process, with $W_{1}$ the time until the first claim occurs, still independent of $\left\{W_{2}, W_{3}, \ldots\right\}$ and $\left\{X_{i}: i \in \mathbf{N}^{+}\right\}$but with a (possibly) different p.f. $k_{1}(t)$. As a special case of the delayed model, the stationary renewal risk model can be defined if the p.f. $k_{1}(t)=\frac{\bar{K}(t-1)}{E\left(W_{2}\right)}, t \in \mathbf{N}^{+}$.

Received July 13, 2009; Revised February 28, 2010.

2010 Mathematics Subject Classification. 91B30, 62P05.

Key words and phrases. discrete time delayed renewal risk model, discounted proper distribution of the deficit, probability generating function of ruin time. 
Further, if $k_{1}(t)=k(t)$, then the delayed renewal risk model becomes the ordinary renewal risk model.

Given the ordinary discrete time renewal model, Li [6] derives a recursive formula for the discounted penalty function with claim waiting times having a discrete $K_{m}$ distribution. In a subsequent paper of Li [7], the discounted penalty function is explicitly expressed in terms of a compound geometric distribution function, and the explicit expressions for the probability generating function (p.g.f.) of the time of ruin, the joint and marginal distributions of the surplus before ruin, the deficit at ruin, the claim causing ruin, as well as their moments are derived. $\mathrm{Wu}$ and $\mathrm{Li}[12]$ obtain a recursive formula satisfied by the penalty function for the discrete renewal risk model with arbitrary interclaim times. Cossette et al. [2] consider a discrete time renewal risk model with premium rate $c \in \mathbf{N}^{+}$. Under the framework of this extension, they investigate the aggregate claim amount process and both finite-time and infinite-time ruin probabilities. They also derive an upper bound and an asymptotic expression for the infinite-time ruin probabilities. See also Cheng et al. [3] and Li and Garrido [8] for related analysis.

In recent years, the delayed renewal model has drawn much attention of researchers. Willmot [10] considers a class of continuous time delayed renewal risk process with a special choice for $k_{1}(t)$, which generalizes both the equilibrium density and the exponential density. Along the similar lines with Willmot [10], Bao and Ye [1] study the delayed renewal risk model with random premium income, the expected discounted penalty function in the delayed renewal model is expressed in terms of the corresponding penalty function in the ordinary renewal model. Willmot and Lin [11, section 11] deal with the ruin probabilities for the delayed and equilibrium renewal risk processes, among many others.

For the discrete time stationary risk model, Pavlova and Willmot [9] express the expected discounted penalty function in terms of the corresponding penalty function in the ordinary renewal risk model. The results for ruin probabilities obtained in Cossette et al. [2] can also be easily generalized to the delayed renewal risk model. There are no further results for the discrete time delayed renewal risk model.

Comparing with the ultimate ruin probability, there are few literatures to deal with the severity of ruin, which is an important quantity in risk theory. The purpose of this paper is to study the discounted proper deficit for the discrete time delayed renewal model in some detail. We will examine what will happen when the d.f. of the proper deficit is asymptotic in the initial surplus $u$. It is not straightforward to take the limit of $u$ from the proper deficit defined below. Therefore, we establish several lemmas to give the upper bounds and asymptotic estimations for the p.g.f. of the ruin time in the ordinary and delayed renewal risk model respectively, which allow for using the dominated convergence theorem in the proof of the main result. As mentioned by Pavlova and Willmot [9], there are some technical details which are somewhat different in the discrete model. In what follows we can see that how the p.g.f. can be 
used to analyze the time of ruin and associated ruin quantities. The results obtained will fill a gap in the scant literature on discrete time renewal risk model.

The rest of this paper is organized as follows. In Section 2, we express the expected discounted penalty function in the delayed renewal risk model in terms of the corresponding discounted penalty function in the ordinary renewal risk model and present the main result. Section 3 establishes some lemmas which are of independent interest on their own right, and Section 4 gives the proof of the main result.

\section{Preliminaries and main result}

For the delayed renewal risk model, let $T_{d}=\min \left\{n \in \mathbf{N}^{+} ; U(n)<0\right\}$ be the time of ruin with $T_{d}=\infty$ if ruin does not occur. If ruin occurs, $\left|U\left(T_{d}\right)\right|$ is the deficit at ruin and $U\left(T_{d}-1\right)$ is the surplus immediately prior to ruin. For $v \in(0,1]$, denote by

$$
m_{v}^{d}(u)=E\left[v^{T_{d}} \omega\left(U\left(T_{d}-1\right),\left|U\left(T_{d}\right)\right|\right) I\left(T_{d}<\infty\right) \mid U(0)=u\right],
$$

the Gerber-Shiu expected discounted penalty function, which is introduced by Gerber and Shiu [4]. Where $\omega(x, y): \mathbf{N} \times \mathbf{N}^{+} \rightarrow \mathbf{N}$ is a nonnegative penalty function of the surplus prior to ruin and the deficit at ruin and $I(\cdot)$ is the indicator function.

Consider $f_{3}^{d}(i, j, t \mid u)=P\left\{U\left(T_{d}-1\right)=i,\left|U\left(T_{d}\right)\right|=j, T_{d}=t \mid U(0)=u\right\}$, $i \in \mathbf{N}, j \in \mathbf{N}^{+}$, the joint p.f. of the surplus just before ruin, deficit at ruin and ruin time. And we define $f_{2}^{d}(i, j \mid u)=\sum_{t=1}^{\infty} v^{t} f_{3}^{d}(i, j, t \mid u)$ as the discounted joint p.f. of $U\left(T_{d}-1\right)$ and $\left|U\left(T_{d}\right)\right|$.

Throughout the entire paper, unless otherwise stated, we use the same notations by omitting the argument $d$ to denote the corresponding quantities in the ordinary renewal model. For example, if $T$ is the time of ruin in the ordinary risk model, then the corresponding penalty function is denoted by

$$
m_{v}(u)=E\left[v^{T} \omega(U(T-1),|U(T)|) I(T<\infty) \mid U(0)=u\right] .
$$

Since the delayed renewal risk process behaves like the ordinary renewal risk process upon the occurrence of the first claim, we obtain the following equation by conditioning on the first drop in surplus below its initial level $u$

$$
\begin{aligned}
m_{v}^{d}(u)= & \sum_{j=1}^{u} \sum_{i=0}^{\infty} \sum_{t=1}^{\infty} v^{t} m_{v}(u-j) f_{3}^{d}(i, j, t \mid 0) \\
& +\sum_{j=u+1}^{\infty} \sum_{i=0}^{\infty} \sum_{t=1}^{\infty} v^{t} \omega(i+u, j-u) f_{3}^{d}(i, j, t \mid 0) \\
= & \sum_{j=1}^{u} m_{v}(u-j) g_{v}^{d}(j)+\sum_{j=u+1}^{\infty} \sum_{i=0}^{\infty} \omega(i+u, j-u) f_{2}^{d}(i, j \mid 0), u \in \mathbf{N}^{+},
\end{aligned}
$$


with

$$
m_{v}^{d}(0)=\sum_{j=1}^{\infty} \sum_{i=0}^{\infty} \omega(i, j) f_{2}^{d}(i, j \mid 0)
$$

where $g_{v}^{d}(j):=\sum_{i=0}^{\infty} f_{2}^{d}(i, j \mid 0)$. The function $g_{v}^{d}$ is a defective distribution function and it plays a very important role in what follows. For notational convenience, we denote $\overline{G_{v}^{d}}(x)=\sum_{j=x+1}^{\infty} g_{v}^{d}(j)$.

By the equation (1), we can obtain a simple relationship between the discounted defective survival distribution of the deficit $\phi_{v}^{d}(u, y)$ in the delayed model and $\phi_{v}(u, y)$ in the ordinary renewal model as follows:

$$
\begin{aligned}
\phi_{v}^{d}(u, y) & :=E\left[v^{T_{d}} I\left(\left|U\left(T_{d}\right)\right|>y\right) I\left(T_{d}<\infty\right) \mid U(0)=u\right] \\
& =\sum_{j=1}^{u} \phi_{v}(u-j, y) g_{v}^{d}(j)+\overline{G_{v}^{d}}(u+y), u \in \mathbf{N}^{+}, y \in \mathbf{N},
\end{aligned}
$$

with $\phi_{v}^{d}(0, y)=\overline{G_{v}^{d}}(y)$.

Furthermore, with $y=0$ in the equation (2) we get the p.g.f. of ruin time $T_{d}$ with respect to discount factor $v$

$$
\begin{aligned}
\phi_{v}^{d}(u)=\phi_{v}^{d}(u, 0) & :=E\left[v^{T_{d}} I\left(T_{d}<\infty\right) \mid U(0)=u\right] \\
& =\sum_{j=1}^{u} \phi_{v}(u-j) g_{v}^{d}(j)+\overline{G_{v}^{d}}(u), u \in \mathbf{N}^{+},
\end{aligned}
$$

with $\phi_{v}^{d}(0)=\overline{G_{v}^{d}}(0)$.

For any p.f. $l$, we will use $\hat{l}(s)$ to denote the corresponding p.g.f. throughout the entire paper. Now we consider the following generalized version of Lundberg equation:

$$
\hat{k}(v / s) \hat{f}(s)=1 .
$$

It is easy to see that the equation (4) has one root greater than 1 . Hence denote by $\xi_{v}$, which can be called a generalized adjustment coefficient as $\mathrm{Li}$ [6]. Wu and $\mathrm{Li}[12]$ show that the generalized Lundberg equation (4) has the same roots as the equation $\hat{g_{v}}(s)=1$, where $g_{v}$ is a defective distribution in the scenario of ordinary renewal model (corresponding to the function $g_{v}^{d}$ ). Similarly, we also adopt the notation $\overline{G_{v}}(x)=\sum_{j=x+1}^{\infty} g_{v}(j)$ for convenience.

In what follows the discounted proper distribution function of the deficit, conditioning on ruin occurring, is defined by $H_{v, u}^{d}(y)=1-\overline{H_{v, u}^{d}}(y)$ where $\overline{H_{v, u}^{d}}(y)=\frac{\phi_{v}^{d}(u, y)}{\phi_{v}^{d}(u)}$. In this short communication we will establish an asymptotic result (as the initial surplus $u \rightarrow \infty$ ) for $\overline{H_{v, u}^{d}}(y)$. 
Theorem 2.1. For $y \in \mathbf{N}$, the asymptotic distribution of the discounted proper deficit in the discrete time delayed renewal risk model is

$$
\lim _{u \rightarrow \infty} \overline{H_{v, u}^{d}}(y)=\frac{\xi_{v}-1}{1-\phi_{v}(0)} \sum_{i=0}^{\infty} \xi_{v}^{i} \bar{G}_{v}(i+y) .
$$

We remark that the asymptotic distribution of $\overline{H_{v, u}^{d}}(y)$ is independent of the distribution of the first interclaim time $W_{1}$. This is because large $u$ implies large $T_{d}$, and as the initial surplus $u$ gets large, the effect of the assumed distribution for the time until the first claim becomes insignificant. In fact, we will see that the asymptotic result for $\overline{H_{v, u}^{d}}(y)$ is of the same form as in the ordinary discrete time renewal risk model. Furthermore, note that in general (5) cannot be solved analytically. However, if we assume the claim waiting times and claim amounts follow some particular distributions, we can get analytical solutions for $\xi_{v}$ and $g_{v}$, then explicit expression for (5) can be obtained. We illustrate this point in the following example.

Example 2.1. We assume that the claim waiting times are shifted negative binomial distributed with $k(t)=t(1-q)^{2} q^{t}, t \in \mathbf{N}^{+}$in the ordinary renewal risk model. Claim amounts are geometrically distributed with $f(x)=(1-$ $\alpha) \alpha^{x-1}, x \in \mathbf{N}^{+}$, and $\hat{f}(s)=s(1-\alpha) /(1-s \alpha)$. Then equation

$$
(s-v q)^{2}(1-s \alpha)-v(1-q)^{2} s^{2}(1-\alpha)=0
$$

has two roots, say $\rho_{1}, \rho_{2}$ with $\left|\rho_{i}\right|<1$, and one root $\xi_{v}>1$. By the equation (56) of $\mathrm{Li}[6]$ we know that

$$
g_{v}(y)=\frac{1-\xi_{v} \alpha}{\xi_{v}} \alpha^{y-1} .
$$

Therefore, the tail distribution of $g_{v}$ satisfies

$$
\bar{G}_{v}(u)=\frac{1-\xi_{v} \alpha}{\xi_{v}(1-\alpha)} \alpha^{u} .
$$

On the other hand, by Theorem 6 of Li [7] we have

$$
\phi_{v}(u)=\frac{1-\xi_{v} \alpha}{\xi_{v}(1-\alpha)} \xi_{v}^{-u} .
$$

Substitution the expressions of $\bar{G}_{v}(u)$ and $\phi_{v}(0)$ into the right side of the equation (5) yields

$$
\lim _{u \rightarrow \infty} \overline{H_{v, u}^{d}}(y)=\alpha^{y} .
$$

\section{Some lemmas}

In this section we will prepare several lemmas before giving the proof of Theorem 2.1. 
Lemma 3.1. For $u \in \mathbf{N}$, the p.g.f. of ruin time $\phi_{v}(u)$ in the ordinary discrete time renewal risk model satisfies the following upper bound:

$$
\phi_{v}(u) \leq \xi_{v}^{-(u+1)} .
$$

Proof. For the ordinary renewal risk model, Li [6] shows that $\phi_{v}(u)$ satisfies the following renewal equation (in an obvious notation)

$$
\phi_{v}(u)=\sum_{j=1}^{u} \phi_{v}(u-j) g_{v}(j)+\overline{G_{v}}(u), u \in \mathbf{N}^{+} .
$$

It is easy to see from (7) that

$$
\hat{\phi}_{v}(s)=\frac{\phi_{v}(0)-\hat{g_{v}}(s)}{(1-s)\left(1-\hat{g_{v}}(s)\right)} .
$$

Equation (8) can be rewritten as

$$
\hat{\phi_{v}}(s)=\frac{1-P_{v}(s)}{1-s},
$$

where $P_{v}(s)=\frac{1-\phi_{v}(0)}{1-\hat{g_{v}}(s)}=\frac{1-\phi_{v}(0)}{1-\phi_{v}(0) Q_{v}(s)}, Q_{v}(s)=\frac{1}{\phi_{v}(0)} \hat{g_{v}}(s)$. The inequality $(6)$ then follows by Lemma 7.2.1 of Willmot and Lin [11] directly.

Lemma 3.2. For $u \in \mathbf{N}$, the asymptotic estimation for the p.g.f. of ruin time $\phi_{v}(u)$ in the ordinary discrete time renewal risk model is:

$$
\phi_{v}(u) \sim \frac{1-\phi_{v}(0)}{\left(\xi_{v}-1\right) \sum_{i=1}^{\infty} i \xi_{v}^{i} g_{v}(i)} \xi_{v}^{-u} .
$$

Proof. From the equation (36) of $\mathrm{Li}[6]$ we can obtain a defective renewal equation for $\phi_{v}(u, y)$ as follows:

$$
\phi_{v}(u, y)=\sum_{j=1}^{u} \phi_{v}(u-j, y) g_{v}(j)+\bar{G}_{v}(u+y), u \in \mathbf{N}^{+} .
$$

Recall that $\hat{g}_{v}\left(\xi_{v}\right)=1$. Then, multiplying (10) by $\xi_{v}^{u}$ yields a standard discrete renewal equation. By the discrete renewal theory (Karlin and Taylor [5]) we have

$$
\lim _{u \rightarrow \infty} \xi_{v}^{u} \phi_{v}(u, y)=\frac{\sum_{i=0}^{\infty} \xi_{v}^{i} \bar{G}_{v}(i+y)}{\sum_{i=1}^{\infty} i \xi_{v}^{i} g_{v}(i)}
$$

Let $y=0$ in $(11)$ we get

$$
\lim _{u \rightarrow \infty} \xi_{v}^{u} \phi_{v}(u)=\frac{\sum_{i=0}^{\infty} \xi_{v}^{i} \bar{G}_{v}(i)}{\sum_{i=1}^{\infty} i \xi_{v}^{i} g_{v}(i)}=\frac{1-\phi_{v}(0)}{\left(\xi_{v}-1\right) \sum_{i=1}^{\infty} i \xi_{v}^{i} g_{v}(i)} .
$$

It is obvious that the equation (9) is equivalent to (12). 
Lemma 3.3. For $u \in \mathbf{N}$, the p.g.f. of ruin time $\phi_{v}^{d}(u)$ in the delayed discrete time renewal risk model satisfies the following upper bound:

$$
\phi_{v}^{d}(u) \leq \frac{\hat{k}_{1}\left(\frac{v}{\xi_{v}}\right)}{\hat{k}\left(\frac{v}{\xi_{v}}\right)} \xi_{v}^{-(u+1)} .
$$

Proof. Conditioning on the time $W_{1}$ and the amount $X_{1}$ of the first claim, we obtain

$$
\phi_{v}^{d}(u)=\sum_{i=1}^{\infty} v^{i}\left[\sum_{j=1}^{u+i} \phi_{v}(u+i-j) f(j)+\bar{F}(u+i)\right] k_{1}(i) .
$$

Note that

$$
\bar{F}(u+i) \leq \xi_{v}^{-(u+i+1)} \sum_{j=u+i+1}^{\infty} \xi_{v}^{j} f(j),
$$

by (6), (14) and (15) we have

$$
\begin{aligned}
\phi_{v}^{d}(u) & \leq \sum_{i=1}^{\infty} v^{i}\left[\sum_{j=1}^{u+i} \xi_{v}^{-(u+i-j+1)} f(j)+\xi_{v}^{-(u+i+1)} \sum_{j=u+i+1}^{\infty} \xi_{v}^{j} f(j)\right] k_{1}(i) \\
& =\xi_{v}^{-(u+1)} \sum_{i=1}^{\infty}\left(\frac{v}{\xi_{v}}\right)^{i}\left[\sum_{j=1}^{\infty} \xi_{v}^{j} f(j)\right] k_{1}(i) \\
& =\xi_{v}^{-(u+1)} \hat{f}\left(\xi_{v}\right) \hat{k}_{1}\left(\frac{v}{\xi_{v}}\right) .
\end{aligned}
$$

Recall that $\xi_{v}$ is the root of the generalized Lundberg equation (4), the inequality (13) follows by (16) immediately.

Lemma 3.4. For $u \in \mathbf{N}$, the following asymptotic result for the p.g.f. of ruin time $\phi_{v}^{d}(u)$ in the delayed discrete time renewal risk model holds:

$$
\phi_{v}^{d}(u) \sim \frac{\hat{k}_{1}\left(\frac{v}{\xi_{v}}\right)}{\hat{k}\left(\frac{v}{\xi_{v}}\right)} \frac{1-\phi_{v}(0)}{\left(\xi_{v}-1\right) \sum_{i=1}^{\infty} i \xi_{v}^{i} g_{v}(i)} \xi_{v}^{-u} .
$$

Proof. Note that the generalized Lundberg equation (4) implies $\hat{f}\left(\xi_{v}\right)<\infty$. Then the inequality $\xi_{v}^{i} \bar{F}(i) \leq \sum_{j=i+1}^{\infty} \xi_{v}^{j} f(j)$ implies that

$$
\lim _{i \rightarrow \infty} \xi_{v}^{i} \bar{F}(i)=0 \text {. }
$$

On the other hand, for any $i \geq j$, by Lemma 3.1 we have

$$
\xi_{v}^{i-j} \phi_{v}(i-j) \leq \frac{1}{\xi_{v}} \leq 1,
$$

then by (12), (18) and bounded convergence theorem we have

$$
\lim _{i \rightarrow \infty} \xi_{v}^{i}\left\{\sum_{j=1}^{i} \phi_{v}(i-j) f(j)+\bar{F}(i)\right\}=\sum_{j=1}^{\infty} \xi_{v}^{j} f(j) \lim _{i \rightarrow \infty} \xi_{v}^{i-j} \phi_{v}(i-j)
$$




$$
=\frac{1}{\hat{k}\left(\frac{v}{\xi_{v}}\right)} \frac{1-\phi_{v}(0)}{\left(\xi_{v}-1\right) \Sigma_{i=1}^{\infty} i \xi_{v}^{i} g_{v}(i)} .
$$

By the equations (14), (19) and use the bounded convergence theorem again, we obtain

$$
\begin{aligned}
& \lim _{u \rightarrow \infty} \xi_{v}^{u} \phi_{v}^{d}(u) \\
= & \lim _{u \rightarrow \infty} \sum_{i=1}^{\infty}\left(\frac{v}{\xi_{v}}\right)^{i} k_{1}(i) \xi_{v}^{u+i}\left[\sum_{j=1}^{u+i} \phi_{v}(u+i-j) f(j)+\bar{F}(u+i)\right] \\
= & \sum_{i=1}^{\infty}\left(\frac{v}{\xi_{v}}\right)^{i} k_{1}(i) \lim _{u \rightarrow \infty} \xi_{v}^{u+i}\left[\sum_{j=1}^{u+i} \phi_{v}(u+i-j) f(j)+\bar{F}(u+i)\right] \\
= & \frac{\hat{k}_{1}\left(\frac{v}{\xi_{v}}\right)}{\hat{k}\left(\frac{v}{\xi_{v}}\right)} \frac{1-\phi_{v}(0)}{\left(\xi_{v}-1\right) \sum_{i=1}^{\infty} i \xi_{v}^{i} g_{v}(i)} .
\end{aligned}
$$

The asymptotic relationship (17) follows from (20) directly.

Lemma 3.5. For $u \in \mathbf{N}$, the tail distribution $\overline{G_{v}^{d}}(u)$ in the delayed discrete time renewal risk model satisfies:

$$
\overline{G_{v}^{d}}(u)=\frac{\phi_{v}^{d}(u)-\sum_{j=1}^{u} \phi_{v}^{d}(u-j) g_{v}(j)-\phi_{v}^{d}(0) \bar{G}_{v}(u)}{1-\phi_{v}(0)} .
$$

Proof. Multiplying both sides of the equation (3) by $s^{u}$ and summing over $u$ from 1 to $\infty$ yields

$$
\hat{\phi}_{v}^{d}(s)=\hat{g_{v}^{d}}(s) \hat{\phi}_{v}(s)+\frac{\phi_{v}^{d}(0)-\hat{g_{v}^{d}}(s)}{1-s} .
$$

Substitute (8) into (22) and multiply by $1-\hat{g_{v}}(s)$ to get

$$
\begin{aligned}
\left(1-\hat{g}_{v}(s)\right) \hat{\phi}_{v}^{d}(s) & =\frac{\phi_{v}^{d}(0)+\phi_{v}(0) \hat{g_{v}^{d}}(s)-\phi_{v}^{d}(0) \hat{g}_{v}(s)-\hat{g_{v}^{d}}(s)}{1-s} \\
& =\phi_{v}^{d}(0) \frac{\phi_{v}(0)-\hat{g}_{v}(s)}{1-s}+\left(1-\phi_{v}(0)\right) \frac{\phi_{v}^{d}(0)-\hat{g}_{v}^{d}(s)}{1-s} .
\end{aligned}
$$

From the equation (23) we get (21) by inverting the p.g.f.

\section{Proof of Theorem 2.1}

Noting that $\hat{g_{v}}\left(\xi_{v}\right)=1$, we have

$$
\lim _{u \rightarrow \infty} \xi_{v}^{u} \bar{G}_{v}(u)=\lim _{u \rightarrow \infty} \xi_{v}^{u} \sum_{j=u+1}^{\infty} g_{v}(j) \leq \lim _{u \rightarrow \infty} \sum_{j=u+1}^{\infty} \xi_{v}^{j} g_{v}(j)=0 .
$$


Since Lemma 3.4 implies that $\lim _{u \rightarrow \infty} \xi_{v}^{u} \phi_{v}^{d}(u)$ is a constant, by (24) and Lemma 3.5 we obtain

$$
\begin{aligned}
\lim _{u \rightarrow \infty} \xi_{v}^{u} \overline{G_{v}^{d}}(u) & =\frac{\lim _{u \rightarrow \infty} \xi_{v}^{u} \phi_{v}^{d}(u)-\lim _{u \rightarrow \infty} \xi_{v}^{u} \sum_{j=1}^{u} \phi_{v}^{d}(u-j) g_{v}(j)}{1-\phi_{v}(0)} \\
& =\frac{\lim _{u \rightarrow \infty} \xi_{v}^{u} \phi_{v}^{d}(u)\left[1-\sum_{j=1}^{\infty} \xi_{v}^{j} g_{v}(j)\right]}{1-\phi_{v}(0)} \\
& =0 .
\end{aligned}
$$

The inequality $\xi_{v}^{u} \overline{G_{v}^{d}}(u+y) \leq \xi_{v}^{u} \overline{G_{v}^{d}}(u)$ together with the equation (25) imply that

$$
\lim _{u \rightarrow \infty} \xi_{v}^{u} \overline{G_{v}^{d}}(u+y)=0 .
$$

On the other hand, by Lemma 3.1 we know that

$$
\xi_{v}^{u} \phi_{v}(u, y) \leq \xi_{v}^{u} \phi_{v}(u) \leq \xi_{v}^{-1} \leq 1 .
$$

Multiplying both sides of the equation (2) by $\xi_{v}^{u}$ and taking limit as $u \rightarrow \infty$, by the dominated convergence theorem and (26) we obtain

$$
\begin{aligned}
\lim _{u \rightarrow \infty} \xi_{v}^{u} \phi_{v}^{d}(u, y) & =\sum_{j=1}^{\infty} \xi_{v}^{j} g_{v}^{d}(j)\left\{\lim _{u \rightarrow \infty} \xi_{v}^{u} \phi_{v}(u, y)\right\}+\lim _{u \rightarrow \infty} \xi_{v}^{u} \overline{G_{v}^{d}}(u+y) \\
& =\sum_{j=1}^{\infty} \xi_{v}^{j} g_{v}^{d}(j)\left\{\lim _{u \rightarrow \infty} \xi_{v}^{u} \phi_{v}(u, y)\right\}
\end{aligned}
$$

Since $\overline{H_{v, u}^{d}}(y)=\frac{\phi_{v}^{d}(u, y)}{\phi_{v}^{d}(u)}$, by $(27)$ we have

$$
\lim _{u \rightarrow \infty} \overline{H_{v, u}^{d}}(y)=\frac{\lim _{u \rightarrow \infty} \xi_{v}^{u} \phi_{v}^{d}(u, y)}{\lim _{u \rightarrow \infty} \xi_{v}^{u} \phi_{v}^{d}(u, 0)}=\frac{\lim _{u \rightarrow \infty} \xi_{v}^{u} \phi_{v}(u, y)}{\lim _{u \rightarrow \infty} \xi_{v}^{u} \phi_{v}(u, 0)} .
$$

We complete the proof of Theorem 2.1 by considering (11), (12) and (28).

Acknowledgements. The authors are very grateful to the anonymous referee for the comments and suggestions which led to an improved presentation of the manuscript. This research was supported by National Natural Science Foundation of China (11001114) and Science Research Foundation of Educational Department of Liaoning Province of China (NO. 2009A423).

\section{References}

[1] Z. Bao and Z. Ye, The Gerber-Shiu discounted penalty function in the delayed renewal risk process with random income, Appl. Math. Comput. 184 (2007), no. 2, 857-863.

[2] H. Cossette, D. Landriault, and E. Marceau, Ruin probabilities in the discrete time renewal risk model, Insurance Math. Econom. 38 (2006), no. 2, 309-323.

[3] S. Cheng, H. U. Gerber, and E. S. W. Shiu, Discounted probabilities and ruin theory in the compound binomial model, Insurance Math. Econom. 26 (2000), no. 2-3, 239-250.

[4] H. U. Gerber and E. S. W. Shiu, On the time value of ruin, N. Am. Actuar. J. 2 (1998), no. $1,48-78$. 
[5] S. Karlin and H. M. Taylor, A First Course in Stochastic Processes, Academic Press, New York, 1975.

[6] S. Li, On a class of discrete time renewal risk models, Scand. Actuar. J. 2005 (2005), no. $4,241-260$.

[7] _ Distributions of the surplus before ruin, the deficit at ruin and the claim causing ruin in a class of discrete time risk models, Scand. Actuar. J. 2005 (2005), no. 4, 271284.

[8] $\mathrm{S} . \mathrm{Li}$ and J. Garrido, On the time value of ruin in the discrete time risk model, Working paper 02-18, Business Economics, University Carlos III of Madrid, 2002.

[9] K. P. Pavlova and G. E. Willmot, The discrete stationary renewal risk model and the Gerber-Shiu discounted penalty function, Insurance Math. Econom. 35 (2004), no. 2, 267-277.

[10] G. E. Willmot, A note on a class of delayed renewal risk processes, Insurance Math. Econom. 34 (2004), no. 2, 251-257.

[11] G. E. Willmot and X. Lin, Lundberg Approximations for Compound Distributions with Insurance Applications, Lecture Notes in Statistics, 156. Springer-Verlag, New York, 2001.

[12] X. Wu and $\mathrm{S} . \mathrm{Li}$, On the discounted penalty function in a discrete time renewal risk model with general interclaim times, Scand. Actuar. J. 2009 (2009), no. 4, 281-294.

ZHEN-HUA BAO

School of Mathematics

LiAONING NORMAL UNIVERSITY

DaLian 116029, P. R. China

E-mail address: zhhbao@126.com

JING WANG

SCHOOL OF Mathematics

Liaoning Normal University

Dalian 116029, P. R. China

E-mail address: wjlnnu@126.com 